\title{
Formation System of Mobile Robot Based on Vision Location
}

\author{
Junfeng Shuai, a ${ }^{1,}$ Feng $\mathrm{Yu}^{2, \mathrm{~b}}$, Bingbing $\mathrm{Yan}^{2, \mathrm{c}}$ and Xiaoming Feng ${ }^{2, \mathrm{~d}}$ \\ School of Mechanical Engineering, Jiamusi University, Jiamusi154007, China \\ a454415020@qq.com, b7792222180@qq.com, cyanbingbing@126.com, d1159527506@qq.com
}

Corresponding author: Bingbing Yan

Keywords: mobile robot, global localization, formation strategy.

\begin{abstract}
In this paper, a formation system of mobile robot based on vision location is designed. The system is composed of intelligent mobile robot, vision location computation, wireless communication system and formation control strategy. The global localization of the mobile robot is carried out by the camera, the circles of different sizes in the top of mobile robot are identified by computer, the image coordinates of each mobile robot are obtained and the number of the image coordinates of each mobile robot is given. After the coordinates and the number of each vehicle are obtained by the leader robot, the rest of the mobile robots are arranged in a corresponding shape by using wireless communication systems and formation control strategy.
\end{abstract}

\section{Introduction}

Different robots need to be identified in the formation system of mobile robots, and the current position of the robot can be acquired, so that the current information of each robot can be returned in time and control the cooperation. Vision based approach has become the most popular method because of its wide adaptation to the environment. In this paper, the formation system of mobile robot based on vision location is composed of intelligent mobile robot, vision location computation, wireless communication system and formation control strategy. The system is fast and reliable.

\section{Scheme Design of Formation System for Mobile Robot Based on Vision Location}

\subsection{System Scheme Overview.}

The overall structure of formation system for mobile robot based on the vision location is shown in Figure 1. The system hardware is composed of intelligent mobile robot, PC and global location camera. Communication between the host computer and the robot, the robot and robot is realized by wireless communication system of CC1101. The image information of robot is collected by global location camera and transmits it to PC location algorithm for processing, the location information is sent to the leader robot through wireless network, according to the control algorithm which is written to the controller module and the formation control strategy to control the rest of mobile robots.

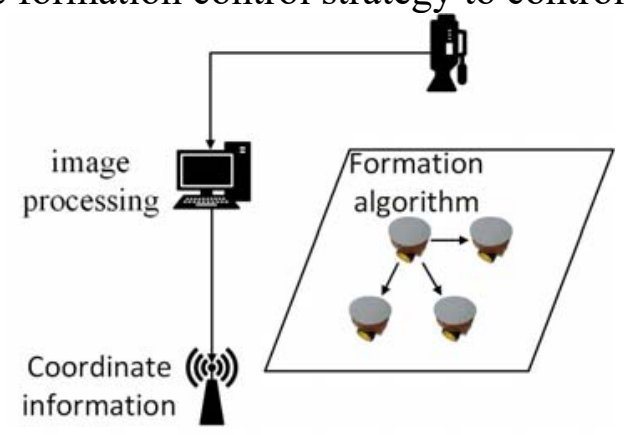

\subsection{The Design of Image Acquisition and Location Module.}

Fig. 1 Overall configuration diagram

Image acquisition and location module is the premise of realizing the coordinated control algorithm for mobile robots. In this paper, global location method based on vision is used to realize the location of the robot. Experimental platform is $3 \mathrm{~m} * 3 \mathrm{~m}$ square area, the camera is mounted on the 
platform to collect the image information of the platform, PC is connected with the camera and the video information is received, the location software of multi mobile robot is run in PC based on OpenCV and VS2010, the circle of different sizes for the mobile robot are recognized to achieve the number and location of real-time for target. The recognition algorithm is shown in Figure 2.

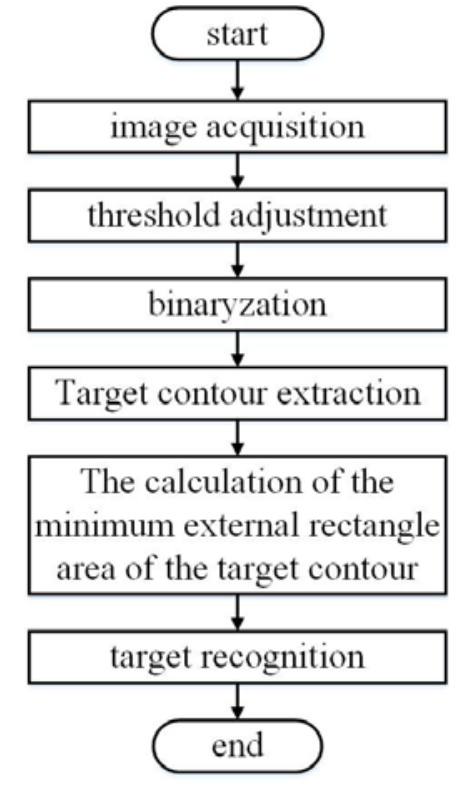

Fig. 2 Recognition algorithm flowchart

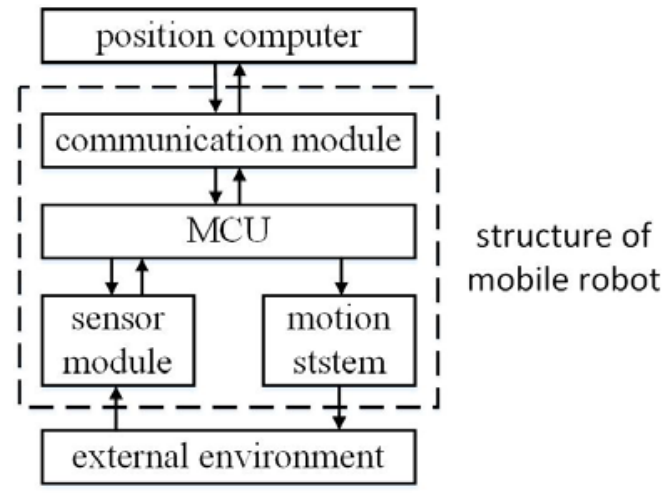

Fig. 3 Mobile Robot Chart

\subsection{The Design of Mobile Robot.}

The mobile robot is composed of model body, controller module, sensor module and communication module. The modules cooperate with each module to realize the formation in the process of system operation.

The function of the controller module is to receive the position of the mobile robot and the environment information, make the information decision and issue the control instruction. The STC89C52 controller is used for mobile robots, the position information of the mobile robot which is obtained by the location computer and the obtained various sensor data, the information is processed by the controller and sends out instructions to guide the movement of the robot. The control algorithm of leader robot controller is shown in Figure 3.

The function of the sensor module is to obtain the environment information around the robot, including the ultrasonic module, the compass module and the mileage meter module. The robot is in the process of running the current coordinates to the target coordinates, the ultrasonic module is used to measure the relative distance between the robot and the other mobile robot when the robot motion path cross; When the local magnetic direction and the image are unified, the compass module is used for measuring the attitude of the current vehicle and is convenient for controlling the direction of the robot; after the image coordinate system is transformed into the coordinate system of experimental platform, the mileage meter module is used to control the running distance of the trolley.

The function of the communication module is to realize the data transmission between the robot and the robot, the PC and the robot, so as to ensure the real-time and accuracy of the data transmission. The CC1101 is used as communication module to achieve signal transmission, the data frame for mat of computer and the leader robot transmission as shown in Figure 4:

\begin{tabular}{|c|c|c|c|c|c|c|}
\hline Start bit & $\begin{array}{c}\text { Robot } \\
\text { number }\end{array}$ & $\begin{array}{c}\mathrm{X} \text { coordinates of } \\
\text { current point }\end{array}$ & $\begin{array}{c}\mathrm{Y} \text { coordinates of } \\
\text { current point }\end{array}$ & $\begin{array}{c}\mathrm{X} \text { coordinates of } \\
\text { target point }\end{array}$ & $\begin{array}{c}\mathrm{Y} \text { coordinates of } \\
\text { target point }\end{array}$ & End bit \\
\hline
\end{tabular}

Fig. 4 The data frame for mat of computer and the leader robot transmission the data frame format of the leader robot and follower robot transmission as shown in Figure 5:

\begin{tabular}{|c|c|c|c|c|c|}
\hline Start bit & $\begin{array}{c}\text { Robot } \\
\text { number }\end{array}$ & $\begin{array}{c}\text { Running } \\
\text { direction }\end{array}$ & $\begin{array}{c}\text { Running } \\
\text { distance }\end{array}$ & $\begin{array}{c}\text { Rumning } \\
\text { speed }\end{array}$ & End bit \\
\hline
\end{tabular}

Fig. 5 The data frame format of the leader robot and follower robot transmission 


\subsection{The Design of Formation Strategy.}

The point strategy mainly is used for the formation strategy. The coordinates of own and the rest of the robot are obtained by the leader robot from the computer, the data is sent to other robots after the calculation, the running direction and distance is given to realize the point motion. A captured frame image from the camera, the coordinate system XOY of global plane is established, Among $\mathrm{S}\left(\mathrm{x}_{0}, \mathrm{Y}_{0}\right)$ is the current coordinate of the mobile robot, $\mathrm{G}\left(\mathrm{x}_{1}, \mathrm{Y}_{1}\right)$ is the target coordinate. As shown in Figure 6.

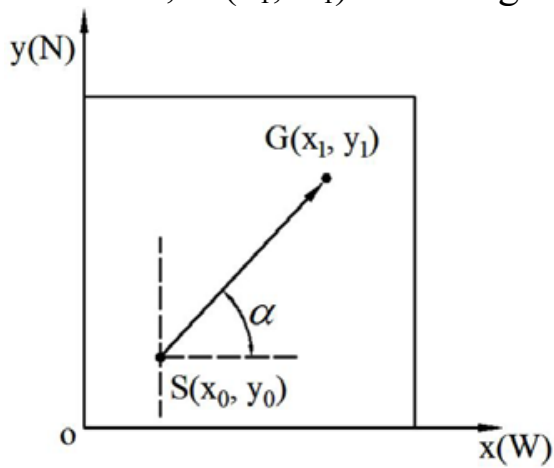

Fig. 6 Experiments schematics

The angle between the movement direction of robot and South Poleis $270^{\circ}-\alpha$, the $\alpha$ can been given as:

$$
\alpha=\arctan \frac{y_{1}-y_{0}}{x_{1}-x_{0}}
$$

themovement distanceof robot can been given as

$$
L=\sqrt{\left(y_{1}-y_{0}\right)^{2}+\left(x_{1}-x_{0}\right)^{2}}
$$

When the angle between the robot motion direction and the South Pole and the distance between them is obtained, the rotation angle of the robot is obtained, and the robot moves along the direction at a certain speed to the target point. In this case, the distance in pixels is converted into the distance $\mathrm{L}$ under the coordinate system of experimental platform.

The formation usually is chosen according to the requirements of the task, the formation is divided into the following four kinds, including row formation, column formation, diamond formation and wedge formation, as shown in Figure 7. It is given by the number of robot moves to the current point corresponding to the number associated with the formation position

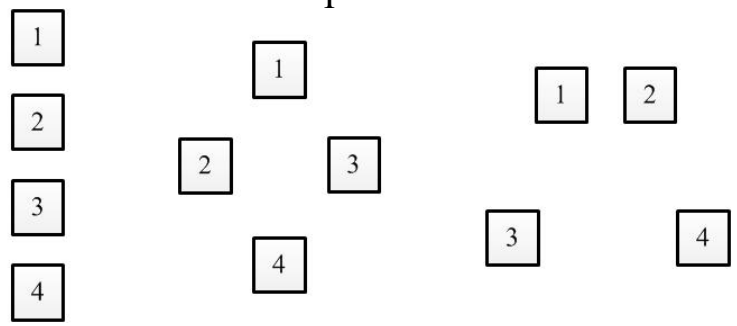

\subsection{Determination of Depth Information.}

Fig. 7 Formation pattern

When the disparity image is obtained by stereo matching, the depth image can be determined and the scene 3D information can be recovered. Generally speaking, the precision of distance measurement is proportional to the matching precision, and is inversely proportional to the length of the base line. The depth measurement accuracy can be improved by increasing the length of the baseline, but it can also the difference between the images and the difficulty of matching. Therefore, all aspects of the factors must be considered to stereo vision system can be accurate designed and each link with a high accuracy can be ensured. 


\section{System Experiment}

According to the demand of the formation, the formation system software of mobile robots based on vision location is developed by using OpenCV library in the VS2010 platform, Robot point experiment is carried out by the formation system of the robot. Different sizes of white circle are put above the robot in the experiment, the robot with a circular surface is captured by using camera, and the numbers are arranged according to the smallest outer rectangle of white circle contour, the formation position is arrived according to point strategy as shown in Figure 8. According to the position coordinates of the robot and the target point, the initial rotation angle is obtained based on fixed point strategy. The movement toward the target point is carried out by rotating the body, own position is adjusted through position information of itself and the target point until reach the target point.

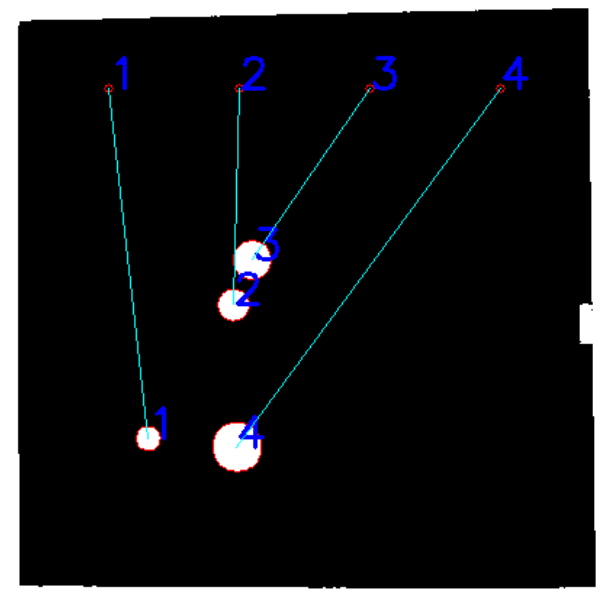

Fig. 8 The robot to the designated test

\section{Summary}

A formation system of mobile robot based on vision location is designed in this paper, the formation system is established, the formation system software of mobile robots based on vision location is developed by using OpenCV library in the VS2010 platform. The numbers are arranged according to the sizes of outer rectangle of white circle contour, the point strategy is put forward and the formation action of robot is realized by the mode of master-slave communication between the robots. In this paper, the global vision location algorithm, communication system and formation strategy are studied, but some problems still need to be further studied. First, the numbers are arranged based on the sizes of external contour of white circle, the method is simple, but it is sensitive to external disturbance. Second, the STC89C52 MCU is used as the main controller, the disadvantages of limited resources and low processing speed, so the more advanced processor should be adopted to improve the function of the mobile robot.

\section{Acknowledgments}

This work has been supported by the Technological Innovation Project of Jiamusi University Graduate (LM2014_012), the Natural Science foundation of Heilongjiang Province (E201254) and the Personnel Training Project of Jiamusi University (RC2009-040).

\section{References}

[1]. J. Toner, Y. Tu. Flocks, Herds. A Quantitative Theory of Flocking. Physical Review E. Vol. 58 (1998) No. 4, p. 4828-4858.

[2]. H.Levine, W.J.Rappel, I.Cohen. Self-organization in Systems of Self-Propelled Particles. Physical Review E. Vol. 63 (2001) No. 2, p. 017-101. 
[3]. E. Clearesta, A. Widyotriatmo. Augie Widyotriatmo, Suprijanto. Mobile Robot Localization Using Modified Particle Filter. Proceedings of 2013 3rd International Conference on Instrumentation Control and Automation, 2013, p. 161-164.

[4]. Schiele B, Crowley J L. A comparison of position estimation techniques using occupancy grids. Robotics and automous systems. Vol. 12 (1994) No. 2, p. 163-171.

[5]. Lynne E. Parker. On the Design of Behavior-based Multi-robot Teams. Advanced Robotics, Vol. 10 (1996) No. 6, p. 547-578.

[6]. Leonard N E, Fiorelli E. Virtual Leader. Artificial Potentials and Coordinated Control of Groups. Proceedings of the 40th IEEE Conference on Decision and Control, 2001, p. 2968-2973. 